\title{
Phenomenological quark model for baryon magnetic moments and beta decay ratios $\left(G_{A} / G_{V}\right)$
}

\author{
Jerrold Franklin* \\ Department of Physics, Temple University, Philadelphia, PA 19122-6082, USA \\ E-mail:v1357@temple.edu
}

ABstract: Baryon magnetic moments and beta decay ratios $\left(G_{A} / G_{V}\right)$ are calculated in a phenomenological quark model. Non-static effects of pion exchange and some orbital excitation are included. Good agreement with experiment is found for a combined fit to all measured baryon magnetic moments and beta decay ratios. The model predicts an antiquark content for the proton that is consistent with the Gottfried sum rule.

The original static quark model (SQM) made predictions for baryon magnetic moments[1-3] that were in remarkable qualitative agreement with early magnetic moment measurements. However, more accurate measurements of the magnetic moments of the baryon octet differ from the SQM predictions by up to 0.2 nuclear magnetons. Also, the SQM can not be reconciled with the ratio $G_{A} / G_{V}$ of beta decay constants in baryon beta decay.

These quantitative failures of the SQM have generally been attributed to various "nonstatic" effects in the quark model. These non-static effects must break SU(3) symmetry if they are to improve the agreement of magnetic moment predictions with experiment. This can be seen from the disagreement with experiment of the sum rules[琣]

$$
\mu(p)-\mu(n)+\mu\left(\Sigma^{-}\right)-\mu\left(\Sigma^{+}\right)+\mu\left(\Xi^{0}\right)-\mu\left(\Xi^{-}\right)=0 \quad(0.49 \pm .05)
$$

and

$$
\mu(p)+2 \mu(n)+\mu\left(\Xi^{-}\right)-\mu\left(\Xi^{0}\right)=0 \quad(-0.43 \pm .01) .
$$

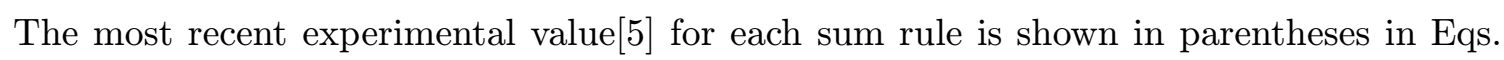
(1) and (2).

For the baryon combinations in each sum rule, the non-static magnetic moment contributions would cancel if the ultimate contribution from each quark were independent of which baryon the quark was in. This "baryon independence" would follow, for instance, if the non-static parts of the baryon wave functions were $\mathrm{SU}(3)$ symmetric. Because of the cancellation of the non-static contributions, it was originally expected that the sum

${ }^{*}$ Speaker. 
rules would be in better agreement with experiment than individual quark moments. However, subsequent tests of the sum rules showed that they disagreed with experiment by more than did any single magnetic moment $[\overline{6} \mid]$. The violation of the sum rules indicates that strong $\mathrm{SU}(3)$ breaking and baryon dependent non-static contributions are required for baryon magnetic moments.

The admixture of pion configurations to the quark model wave functions has been proposed [i] as an important SU(3) breaking non-static effect that would break the sum rules of Eqs. (1) and (2). Such pion contributions were shown to improve quark model magnetic moment predictions significantly. But there was still substantial disagreement with experiment for some of the moments.

In this talk, we show that the inclusion of orbital excitation, along with the pion contribution, permits us to extend the model to simultaneously fit magnetic moments and the beta decay ratios $G_{A} / G_{V}$, along with a better overall agreement with experiment. It had been very difficult to reconcile the quark model magnetic moment predictions with quark model beta decay ratios, especially $G_{A} / G_{V}$ for neutron decay. The combination of the non-static effects (pionic and orbital) now makes it possible with the same quark model to achieve good agreement with experiment for the combined set of baryon magnetic moments and beta decay constants. The detailed calculation of these contributions to

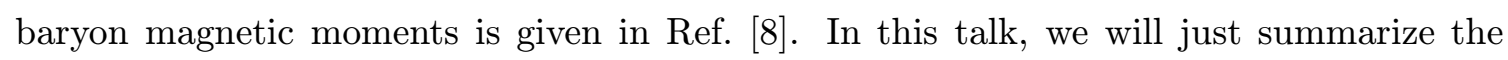
calculation and present results.

There are two kinds of pion contribution. If pions are created and then absorbed by the same quark, they affect only that quark's anomalous moment. This contribution is independent of which quark the baryon is in. This means it cannot affect the magnetic moment sums in Eqs. (1) and (2), and so cannot improve the overall prediction for baryon magnetic moments. The absorption of an emitted charged pion by a different quark in the same baryon leads to exchange currents. These are different for different baryons. For instance, the $u$ quark in a proton can emit a positive pion that is then absorbed by the $d$ quark in the proton. But this type of exchange current cannot occur in a $\Sigma^{+}$hyperon where there is no d quark. Because the pion exchange contributions are baryon dependent, they do affect the sum rules, and can improve the prediction of baryon magnetic moments [9]. Any calculation that imposes isotopic spin conservation at both the quark and baryon level will automatically include these exchange contributions.

If the exchange currents were $\mathrm{SU}(3)$ symmetric, then kaon and eta exchange currents would compensate for the pion exchange currents, preserving the disagreement with experiment of the sum rules. We assume that pion exchange dominates because of the particularly small mass of the pion. The effect of the heavier meson exchanges is neglected, breaking $\mathrm{SU}(3)$ as is necessary to improve agreement with experiment.

We now present the results of a $\chi^{2}$ fit to experiment of eleven magnetic moment predictions and five beta decay ratio predictions. The model predicts quark model magnetic moments and beta decay constants, modified by pion direct and exchange currents, and some orbital excitation. The static static quark model involves two parameters, the input masses of the nucleon and strange quarks. The pion contribution involves three additional parameters, $P_{\pi}$, the percentage of pion admixture in the nucleon, $M$, the effective pion 
magnetic moment, and $R_{\Delta}$, the ratio of $\Delta-\pi$ to $N-\pi$ admixture in the nucleon. The orbital contribution is characterized by the probability $\eta$ of the orbital excitation. So that we are fitting sixteen experimental quantities with six parameters, corresponding to ten degrees of freedom (DF).

The results of this fit are shown in Table 1. The pure quark model two parameter fit, and the fit with only the pion contribution are also shown for comparison. We have included the model prediction for the beta decay ratio $G_{A / V}\left(\Delta^{++} \rightarrow p\right)$, which is used in the calculation of weak proton capture on ${ }^{3} H e\left[{ }^{1}\left[\overline{1}{ }^{i}\right]\right.$. The resonance transistion moment $\mu\left(\Delta^{+} p\right)$ is not included in the fit because its experimental determination is not clear. All the magnetic moments are in units of nuclear magnetons $(\mathrm{nm})$, while the beta decay ratios are pure numbers. In determining $\chi^{2}$, we have used a theoretical error of $0.05 \mathrm{~nm}$ added in quadrature with the experimental errors. This is used to avoid having the fit to theory arbitrarily dominated by the most accurate measurements.

In all fits, the $\Lambda$ quark model state has been corrected for $\Lambda-\Sigma^{0}$ mixing [i] $\left.{ }_{1}^{1} \bar{i}\right]$ resulting from electromagnetic and QCD mass dependent isospin breaking in the quark model. This mixing is required in any consistent quark model calculation at this level of accuracy. The mixing adds about $0.04 \mathrm{~nm}$ to the $\Lambda$ magnetic moment, and has a somewhat smaller effect on the other cases involving the $\Lambda$. Mixing is included in all the $\Lambda$ entries shown in Table 1 .

The $\chi^{2}$ fit for the static quark model (SQM) in Table 1 does not include the beta decay constant ratios. It is clear that the SQM is especially bad for the neutron decay, and including it would raise $\chi^{2}$ to well over 100. Among the magnetic moments, the Sigmas and the Xis are the worst fit for the SQM. Including Pion exchange considerably improves the magnetic moment fits. The Sigma problem is corrected, but there is still a mismatch between the $\mathrm{Xi}$ and the nucleon moments. The most remarkable feature of the pion fit is the great improvement in $G_{A / V}$ for the neutron. This permits an overall fit to both beta decay ratios and magnetic moments. But this still is not enough to achieve really good agreement with experiment. Finally, adding the orbital state is seen to achieve a reasonable fit.

The best fit parameters for the (pi+orbital) case are shown at the bottom of table 1 . The \pm values on the parameters correspond to an increase in $\chi^{2}$ of $\chi^{2} / D F$. The parameters all have reasonable values. The probability of pions in the physical nucleon is rather high, but $M$ is close to the orbital magnetic moment for a pion of the physical mass. Although $R_{\Delta}$ is not large, the decuplet cannot be completely left out. Doing so increases $\chi^{2}$ to 32 .

The importance of each effect can be judged by the effect on $\chi^{2}$ when it is left out. Leaving out the orbital excitation $(\eta=0)$ increases $\chi^{2}$ to 51 , while leaving out the pion exchange $\left(P_{\pi}=0\right)$ increases $\chi^{2}$ to 104 . So it is clear that a combination of non-static effects (in this model, pion exchange, decuplet baryons, and orbital excitation) is required to achieve a reasonable fit to all baryon moments and beta decay ratios. That is why so many earlier calculations that concentrated on only one non-static effect could not achieve good overall fits.

The quark and pion wave functions can be used to calculate the quark spin projections $\Delta u, \Delta d$, and the total quark spin projection $\Sigma$. It follows from isotopic spin rotation that 


\begin{tabular}{|c|c|c|c|c|}
\hline & Expt. & SQM & Pion & $\mathrm{Pi}+$ Orbital \\
\hline$\mu(p)$ & 2.79 & $2.75(0.7)$ & $2.65(7.7)$ & $2.69(4.6)$ \\
\hline$\mu(n)$ & -1.91 & $-1.84(1.9)$ & $-2.04(6.7)$ & $-2.00(2.8)$ \\
\hline$\mu\left(\Sigma^{+}\right)$ & $2.46 \pm .01$ & $2.65(14.7)$ & $2.53(2.0)$ & $2.52(1.5)$ \\
\hline$\mu\left(\Sigma^{-}\right)$ & $-1.16 \pm .03$ & $-1.02(6.7)$ & $-1.14(0.2)$ & $-1.18(0.1)$ \\
\hline$\mu\left(\Xi^{0}\right)$ & $-1.25 \pm .01$ & $-1.44(13.7)$ & $-1.42(10.7)$ & $-1.27(0.2)$ \\
\hline$\mu\left(\Xi^{-}\right)$ & $-0.65 \pm .00$ & $-0.52(6.3)$ & $-0.54(4.8)$ & $-0.59(1.5)$ \\
\hline$\mu(\Lambda)$ & $-0.61 \pm .00$ & $-0.67(1.2)$ & $-0.67(1.1)$ & $-0.56(1.0)$ \\
\hline$\mu(\Sigma, \Lambda)$ & $1.61 \pm .08$ & $1.57(0.2)$ & $1.46(2.6)$ & $1.51(1.1)$ \\
\hline$\mu\left(\Omega^{-}\right)$ & $-2.02 \pm .05$ & $-1.87(4.6)$ & $-1.91(2.2)$ & $-2.08(0.6)$ \\
\hline$\mu\left(\Delta^{++}\right)[1 \overline{1} \overline{2}]$ & $6.2 \pm .7$ & $5.50(1.8)$ & $5.49(1.9)$ & $6.17(0.0)$ \\
\hline$\mu\left(\Delta^{+}, \mathrm{p}\right)$ & & 2.59 & 2.49 & 2.79 \\
\hline$G_{A / V}(\mathrm{n}, \mathrm{p})$ & $1.27 \pm .00$ & $1.67(64)$ & $1.33(1.8)$ & $1.33(1.4)$ \\
\hline$G_{A / V}(\Lambda, \mathrm{p})$ & $0.72 \pm .02$ & $1.00(27)$ & $0.86(6.9)$ & $0.77(0.9)$ \\
\hline$G_{A / V}\left(\Xi^{-}, \Lambda\right)$ & $0.25 \pm .05$ & $0.33(1.9)$ & $0.30(0.6)$ & $0.26(0.0)$ \\
\hline$G_{A / V}\left(\Sigma^{-}, \mathrm{n}\right)$ & $-0.34 \pm .02$ & $-0.33(0.0)$ & $-0.30(0.4)$ & $-0.21(6.1)$ \\
\hline$G_{A / V}\left(\Xi^{0}, \Sigma^{+}\right)\left[\begin{array}{l}1 \\
1\end{array}\right.$ & $1.24 \pm .27$ & $1.67(6.0)$ & $1.53(1.1)$ & $1.38(0.3)$ \\
\hline$G_{A / V}\left(\Delta^{++}, \mathrm{p}\right)$ & & -1.63 & -2.09 & $-2.08 \pm .06$ \\
\hline$\chi^{2}-D F$ & & $52-8$ & $51-11$ & $22-10$ \\
\hline$m_{u}(\mathrm{MeV})$ & & 340 & 340 & $298 \pm 20$ \\
\hline$m_{s}(\mathrm{MeV})$ & & 500 & 490 & $452 \pm 20$ \\
\hline$P_{\pi}$ & & 0 & $29 \%$ & $33 \pm 7 \%$ \\
\hline$M(\pi)(\mathrm{nm})$ & & & 4.8 & $4.8 \pm 1.0$ \\
\hline$R_{\Delta}$ & & & $3 \%$ & $11 \pm 5 \%$ \\
\hline$\eta($ orbital $)$ & & 0 & 0 & $8 \pm 2 \%$ \\
\hline
\end{tabular}

Table 1: Fit of the quark model with pion and orbital contributions. Experimental values are from Ref. [5], except where noted otherwise.

the quark spin projections are related to $G_{A / V}$ for the neutron by

$$
G_{A / V}(n \rightarrow p)=\Delta u-\Delta d
$$

It has to be emphasized here that these quark spin projections are for the proton in its rest system. They are not the same as corresponding quark spin projections on the light cone at infinite momentum, which are calculated using QCD sum rules for polarized deep inelastic scattering asymmetries. Since QCD is a strong interaction, a boost to infinite momentum produces gluons and quark-antiquark pairs that were not in the rest frame wave function. This changes the individual and total quark spin projections. Equation (3ii) is not affected by the boost if it is assumed that the quark pairs produced in the boost are charge symmetric. It then becomes the well known Bjorken sum rule.

We find for the rest frame spin projections

$$
\Delta u=0.98 \pm .05, \quad \Delta d=-0.35 \pm .01, \quad \Sigma=0.63 \pm .06 .
$$


While this shows a considerable decrease in total quark spin projection from the static quark model value $\Sigma=1$, it is not as great a decrease as that indicated in QCD sum rules. Note that, since this model has no SU(3) symmetry, $\Delta s=0$.

Considering the pions as quark-antiquark pairs, we can also calculate the antiquark content $\bar{u}$ and $\bar{d}$ of the proton. We find

$$
\bar{u}=0.07, \quad \bar{d}=0.26, \quad \bar{d}-\bar{u}=0.19 \pm .04 .
$$

With this value for $\bar{d}-\bar{u}$, the quark and antiquark contribution to the Gottfried sum rule[i] $[\overline{4}]$ is

$$
S_{G}=\frac{1}{3}[1-2(\bar{d}-\bar{u})]=0.21 \pm .03,
$$

in good agreement with the experimental result [1] $\left.\overline{1}_{-1}\right]$ of $S_{G}=0.24 \pm .01$. This prediction would survive a boost because the quark pairs produced by QCD are expected to have equal numbers of $u-\bar{u}$ and $d-\bar{d}$ pairs.

Our main conclusion is that a relatively simple phenomenological quark model can provide a combined fit to the beta decay ratios and magnetic moments. The longstanding problem of reducing the static quark model prediction of $5 / 3$ for the neutron $G_{A / V}$ can be solved if there is a sizeable pion component in the nucleon, along with some orbital and decuplet excitation. The pions in the proton wave function also provide the appropriate difference of $\bar{d}-\bar{u}$ antiquarks to satisfy the Gottfried sum rule.

\section{References}

[1] G. Morpurgo, 'Pिhysica $\mathbf{2}(\overline{1} \overline{9} 6 \overline{5}) \overline{9} \overline{5}$

W. Thirring, 'Acta Phys. A Astriaca, Suppl.

[2] H. R. Rubinstein, F. Scheck, and R. Socolow, 'Phys. Rev. 154 (1967) 1608'

[3] J. Franklin, 'Phys.

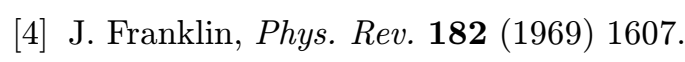

[5] Particle Data Group, C. Caso et al., 'Eur. Physs. $\bar{J}$. $\mathbf{3}(\overline{1} \overline{9} \overline{8})$ 1.'.

[6] J. Franklin, 'Phys.Rev. D 20 (1979) 1742' Phys. Rev. D_29 (1984) 2648

[7] J. Franklin, 'Phys. Rev. D $30-198421542$

[8] J. Franklin, hep-ph/0103139i.

[9] J. Franklin, hep-ph/9807407y Phys. Rev. D 61.

[10] L. E. Marcucci et al., 'P

[11] N. Isgur, 'P

J. Franklin, D. B. Lichtenberg, W. Namgung, D. Carydas, 'Phys. Rev. D 24 (19 $19 \overline{1}) 2 \overline{9} 10 ;$

G. Karl, 'Phys. Lett. B $\mathbf{3} \overline{2}(\overline{9} 94) 149 ;$

J. Franklin, 1 hys. Rev. D 55 s 1997$) 425$

[12] G. Lopez Castro and A. Mariano, nuc-th/0006031.

[13] The KTeV experiment at Fermilab reported by Nickolas Solomey in hep-ex/001

[14] R. D. Field and R. P. Feynman, 'P

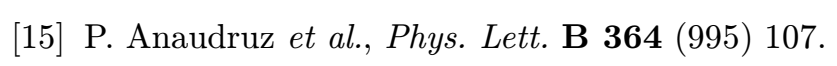

